\title{
Motion compensated beamforming in synthetic aperture vector flow imaging
}

\section{Oddershede, Niels; Jensen, Jørgen Arendt}

\section{Published in:}

Proceedings of the IEEE Ultrasonics Symposium

Link to article, DOI:

10.1109/ULTSYM.2006.490

Publication date:

2006

Document Version

Publisher's PDF, also known as Version of record

Link back to DTU Orbit

\section{Citation $(A P A)$ :}

Oddershede, N., \& Jensen, J. A. (2006). Motion compensated beamforming in synthetic aperture vector flow imaging. In Proceedings of the IEEE Ultrasonics Symposium (pp. 2027-2031). IEEE.

https://doi.org/10.1109/ULTSYM.2006.490

\section{General rights}

Copyright and moral rights for the publications made accessible in the public portal are retained by the authors and/or other copyright owners and it is a condition of accessing publications that users recognise and abide by the legal requirements associated with these rights.

- Users may download and print one copy of any publication from the public portal for the purpose of private study or research.

- You may not further distribute the material or use it for any profit-making activity or commercial gain

- You may freely distribute the URL identifying the publication in the public portal 


\title{
Motion compensated beamforming in synthetic aperture vector flow imaging
}

\author{
Niels Oddershede and Jørgen Arendt Jensen \\ Center for Fast Ultrasound Imaging, Ørsted•DTU, Build. 349, \\ Technical University of Denmark, DK-2800 Lyngby, Denmark
}

\begin{abstract}
In synthetic aperture imaging the beamformed data from a number of emissions are summed to create dynamic focusing in transmit. This makes the method susceptible to motion, which is especially the case for the synthetic aperture flow estimation method, where large movements are expected. In this paper, these motion effects are considered.

A number of Field II simulations of a single scatterer moving at different velocities are performed both for axial and lateral velocities from 0 to $1 \mathrm{~m} / \mathrm{s}$. Data are simulated at a pulse repetition frequency of $5 \mathrm{kHz}$. The signal-to-noise ratio (SNR) of the beamformed response from the scatterer at all velocities is compared to that of a stationary scatterer. For lateral movement, the SNR drops almost linearly with velocity to $-4 \mathrm{~dB}$ at $1 \mathrm{~m} / \mathrm{s}$, while for axial movement the SNR drop is largest, when the scatterer moves a quarter of a wavelength between emissions. Here the SNR is $\mathbf{- 1 0} \mathrm{dB}$ compared to the stationary scatterer.

A 2D motion compensation method for synthetic aperture vector flow imaging is proposed, where the former vector velocity estimate is used for compensating the beamforming of new data. This method is tested on data from an experimental flow rig acquired using our RASMUS experimental ultrasound scanner and a 5.5 MHz linear array transducer. A $11.25 \mu$ s non-linear chirp is used as excitation and the data from 128 emissions is used for estimating the flow direction and magnitude at a profile across the tube.

The measurement was conducted at a flow angle of $60^{\circ}$ with respect to the axial direction and a peak velocity of $0.1 \mathrm{~m} / \mathrm{s}$ sampled at a pulse repetition frequency of $1 \mathrm{kHz}$. The mean bias across the profile was $\mathbf{- 8 . 4 \%}$ with respect to the peak velocity and the mean standard deviation was $\mathbf{1 2 . 2} \%$ prior to compensation. When the proposed compensation was applied a mean bias of $-3.6 \%$ and a mean standard deviation of $2.8 \%$ was seen.
\end{abstract}

\section{INTRODUCTION}

Synthetic transmit aperture (STA) imaging is susceptible to motion artifacts due to the summation of a number of low resolution images created at different time instances. This results in incoherent summation and degradation of both resolution, contrast, and signal-to-noise ratio (SNR). The purpose of this paper is to implement and test a beamforming approach for synthetic aperture vector flow imaging which compensates for 2-D motion.

The effects of tissue motion in synthetic aperture ultrasound have been studied by various authors [1], [2], [3], [4], [5]. Trahey and Nock investigated motion effects in a synthetic receive aperture system in [1] and showed that motion along the axial direction has a far larger effect on the resolution than lateral movement. Also, it was argued that the problem increases with the length of the emission sequence and the center frequency. A 1-D motion compensation method based on axial cross-correlation of low resolution images (LRIs) was proposed. Hazard and Lockwood [3] tested motion effects in a STA system with 3 emissions in the emission sequence. They showed that axial movement on the order of $3 \%$ of the wavelength between emissions had only little effect on the resolution and virtually no effect on the contrast, whereas a 10 times larger movement resulted in both a slight shift and widening of the main-lobe and a reduced contrast.

A method for blood flow velocity estimation using STA was presented in [6]. Here data were beamformed along the axial direction and a cross-correlation estimator was used. The STA velocity estimator of [7] was based on cross-correlation of lines beamformed along the flow, assuming the direction of flow is known.

A method for estimating the direction of flow was presented in [8] based on a search for the highest normalized crosscorrelation as a function of angle. By finding both the inplane flow direction and magnitude, 2-D velocity vectors can be displayed for in principle all image points. The synthetic aperture velocity estimation method as presented in [8] is shortly described in Section II. In these methods the resolution and contrast degradation due to the motion of blood are neglected resulting in a smearing of the point spread function and a decrease in SNR.

In [9] a 2-D motion compensation method for STA Bmode imaging was presented. The method used an interleaved sequence consisting of both a long STA sequence for making high resolution B-mode images and a short STA sequence for motion estimation. The cross-correlation estimator of [8] was used on the short sequence to find the tissue motion in two dimensions, and a compensation was made from the estimated tissue motion, when beamforming the B-mode image from the long sequence.

In this paper the motion compensation method presented in [9] is used in flow estimation. Here only a short sequence is used for both blood velocity estimation and motion compensation. The previous $2-\mathrm{D}$ velocity vector estimate is used for compensating for the motion of the blood in the initial beamforming of the following high resolution lines. This method is described in Section III, simulated in Section IV, and tested on flow phantom data in Section V.

\section{SyNTHETIC APERTURE VELOCITY ESTIMATION}

This section describes how the STA velocity estimation method presented in [8] is performed. Due to the dynamic 


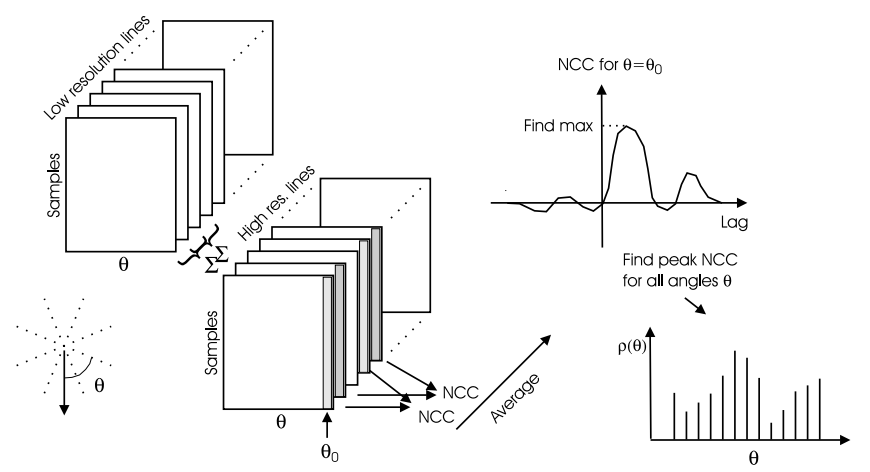

Fig. 1. The synthetic aperture vector flow imaging algorithm. Lines are beamformed in a star-shape as shown in the lower left corner. Low resolution lines are coherently summed to create high resolution lines. After stationary echo cancelling these are cross-correlated and averaged, and the flow angle is estimated as the angle of maximum normalized cross-correlation. The velocity magnitude is found as the cross-correlation peak along this direction.

focus in both transmit and receive, data can be beamformed in every point [7]. This is used for determining the flow direction and velocity.

For every point $\overrightarrow{p_{0}}$, where the velocity direction and magnitude is estimated, a number of lines $\vec{p}(n, \theta)$ are beamformed through the point at different angles

$$
\vec{p}(n, \theta)=\overrightarrow{p_{0}}+\Delta x[\sin \theta, 0, \cos \theta]\left(n-\frac{N}{2}\right),
$$

where $n=[0,1, \ldots, N]$ is the sample index of the lines, $\theta=$ $\left[0, \Delta \theta, 2 \Delta \theta, \ldots, 180^{\circ}\right]$ are the angles with respect to the beam axis $z$, and $\Delta x$ is the spatial sampling distance. Notice, a 3-D right-hand cartesian coordinate system is used where $x$ follows the lateral direction, $y$ the elevation direction, and $z$ the axial, while the origo is placed at the center of the transducer surface. The lines in (1) create a star-shape through the point $\overrightarrow{p_{0}}$ as shown in the bottom left of Fig. 1. The low resolution lines are beamformed by delaying the received signals from each of the receiving elements according to the time of flight and summing over all $Q$ receiving elements as

$$
l_{k}(n, \theta)=\sum_{q=0}^{Q-1} r_{k, q}\left(t_{k, q}(\vec{p}(n, \theta))\right)
$$

where $r_{k, q}(t)$ is the signal received at element $q$ at emission $k$ and $t_{k, q}(\vec{p}(n, \theta))$ is the time of flight from the virtual source used at emission $k$ to the point $\vec{p}(n, \theta)$ and back to receiving element $q$ given by

$$
t_{k, q}(\vec{p}(n, \theta))=\frac{\left\|\vec{p}(n, \theta)-\vec{r}_{x m t, k}\right\|+\left\|\vec{r}_{r c v, q}-\vec{p}(n, \theta)\right\|}{c},
$$

where $\vec{r}_{x m t, k}$ is the position of the virtual source used at emission $k, \vec{r}_{r c v, q}$ is the position of receiving element $q$, and $c$ is the speed of sound. The high resolution lines are formed by summing $K$ consecutive low resolution lines, where $K$ is the number of emissions in the emission sequence, as

$$
h_{k}(n, \theta)=\sum_{k^{\prime}=0}^{K-1} l_{k+k^{\prime}}(n, \theta) \text {. }
$$

The flow direction is found by searching for the angle of maximum normalized cross-correlation (NCC) [8]. The NCC between two high-resolution lines acquired using the same emission sequence is given by

$$
\rho_{k}(m, \theta)=\frac{\sum_{n=0}^{N} h c_{k}(n, \theta) h c_{k+K}(n+m, \theta)}{\sqrt{\sum_{n=0}^{N} h c_{k}^{2}(n, \theta) \sum_{n=0}^{N} h c_{k+K}^{2}(n, \theta)}},
$$

where $h c_{k}(n, \theta)$ is the high resolution line after stationary echo cancelling, and $K$ is the number of emissions in the emission sequence. The stationary echo cancelling is done using a linear regression filter [10] over high resolution lines made using the same emission sequence. $L$ cross-correlations are averaged

$$
\rho_{a v}(m, \theta)=\frac{1}{L} \sum_{k=0}^{L-1} \rho_{k}(m, \theta)
$$

and the maximum cross-correlation at each angle is found

$$
\rho(\theta)=\max \left(\rho_{a v}(m, \theta)\right) .
$$

The flow direction is then found as the angle where $\rho$ has its maximum

$$
\theta_{d}=\arg \max _{\theta} \rho(\theta)
$$

The discrete angle estimate $\theta_{d}$ is interpolated using a parabolic approximation given by [11]

$$
\hat{\theta}=\theta_{d}-\frac{\rho\left(\theta_{d}+\Delta \theta\right)-\rho\left(\theta_{d}-\Delta \theta\right)}{2\left(\rho\left(\theta_{d}+\Delta \theta\right)-2 \rho\left(\theta_{d}\right)+\rho\left(\theta_{d}-\Delta \theta\right)\right)} \Delta \theta,
$$

where $\Delta \theta$ is the angular sampling interval. The flow velocity along the estimated direction is then found by repeating the beamforming along $\hat{\theta}$ and finding the lag of maximum correlation

$$
m_{d}=\arg \max _{m} \rho_{a v}(m, \hat{\theta})
$$

This lag can be interpolated using parabolic interpolation to yield $\hat{m}$. The velocity magnitude along $\hat{\theta}$ is then found by

$$
\hat{v}_{\hat{\theta}}=\frac{\hat{m} \Delta x f_{p r f}}{K}
$$

where $f_{p r f}$ is the pulse repetition frequency. By applying prior knowledge of the expected velocity range, the search range can be limited by only evaluating the cross correlation function (5) in lags corresponding to the expected velocity range.

\section{VELOCITY ESTIMATION WITH MOTION COMPENSATION}

To sum low resolution lines (LRLs) in phase during movement, a compensation can be made for the current velocity during beamformation. In a velocity estimation system the velocity is of course not known in advantage, but by assuming a constant velocity, the velocity vector estimate from the latest time instance $\vec{v}_{e s t}$ can be used as a compensation for creating the next high resolution lines (HRLs). The assumption of constant velocity is rarely fulfilled in-vivo but due to the continuous availability of data in STA and the recursive 


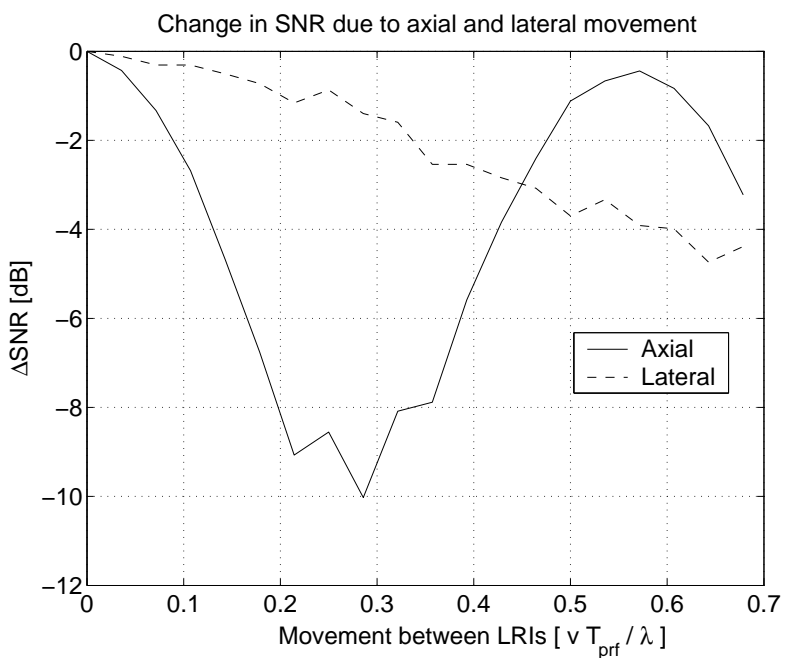

Fig. 2. The change in SNR due to axial and lateral motion. Found by simulation using a non-linear chirp sweeping frequencies from 3.7 to 7.3 $\mathrm{MHz}$.

formation of HRLs, the velocity can be closely tracked. The motion compensated HRLs are formed as

$$
h_{k}(n, \theta)=\sum_{k^{\prime}=0}^{K-1} \sum_{q=0}^{Q-1} r_{k+k^{\prime}, q}\left(t_{k+k^{\prime}, q}\left(\vec{p}(n, \theta)+k^{\prime} \vec{v}_{\text {est }} / f_{p r f}\right)\right) \text {, }
$$

where $\vec{v}_{\text {est }}=\hat{v}_{\hat{\theta}}[\sin (\hat{\theta}), 0, \cos (\hat{\theta})]$ is the latest velocity vector estimate and $f_{p r f}$ is the pulse repetition frequency. The motion effect will be fully compensated for if $\vec{v}_{\text {est }}$ equals the true velocity. Cross-correlating two high-resolution lines $K$ emissions apart will still expectedly peak at the lag corresponding to the blood velocity. Even if an erroneous velocity estimate is used for the compensation, the point spread function of a single scatterer is simply smeared, which was already the case prior to compensation.

\section{Simulations}

A number of simulations were performed using Field II [12], [13]. Here a single scatterer, initially located at depth $z=30 \mathrm{~mm}$ and below the aperture center $x=0 \mathrm{~mm}$, is moving along the axial direction, while $K=8$ low resolution images (LRIs) are acquired. The high resolution image (HRI) is created by summing the LRIs. The SNR of the HRI is then compared to the SNR of the HRI assuming no motion. The simulation was repeated for velocities of $v^{\prime}=0,5,10, \ldots, 95$ $\mathrm{cm} / \mathrm{s}$ corresponding to movement of up to $0.68 \lambda$ between each LRI, where $\lambda=c / f_{0}$ is the wavelength. The excitation waveform was an $11.25 \mu$ s non-linear chirp designed using a frequency sampling method [14]. The parameters used in the simulation are given in Table I.

For each of the simulations the change in SNR due to movement $\triangle S N R$ is found as

$$
\Delta S N R=10 \log _{10}\left(\frac{E_{v=v^{\prime}}}{E_{v=0}}\right)
$$

TABLE I

PARAMETERS USED FOR SIMULATION AND MEASUREMENT.

\begin{tabular}{ll}
\hline Transducer & \\
\hline Transducer type & Linear array \\
Number of transducer elements & 128 \\
Transducer element pitch & $0.3 \mathrm{~mm}$ \\
Transducer element kerf & $0.035 \mathrm{~mm}$ \\
Transducer element height & $5 \mathrm{~mm}$ \\
Elevation focus & $20 \mathrm{~mm}$ \\
Center frequency, $f_{0}$ & $5.5 \mathrm{MHz}$ \\
Wavelength, $\lambda=c / f_{0}$ & $0.28 \mathrm{~mm}$ \\
\hline STA transmission & \\
\hline Excitation type & $11.25 \mu \mathrm{s} \mathrm{non-linear} \mathrm{chirp}[14]$ \\
Frequencies swept & $\sim 3.5-7.5 \mathrm{MHz}$ \\
Number of emitting elements & 11 \\
Number of receiving elements, $Q$ & 64, centered at emission \\
Number of emissions in sequence, $K$ & 8 \\
Number of virtual sources, $(=K)$ & 8 \\
Virtual source positions & {$\left[x_{v s}, 0,-2.5\right] \mathrm{mm}$} \\
Pulse repetition frequency, $f_{p r} f$ & $5 \mathrm{kHz}($ sim.) and $1 \mathrm{kHz}(\mathrm{meas})$. \\
\hline Simulation, Inter-LRI movement & \\
\hline Velocities simulated, $v^{\prime}$ & $0,5,10, \ldots, 95 \mathrm{~cm} / \mathrm{s}$ \\
Corresponding inter-LRI movement & $0, \ldots, 0.68 \lambda$ \\
\hline Measurement, Flow rig setup & \\
\hline Tube radius, $R$ & $6 \mathrm{~mm}$ \\
Flow angles, $\theta$ & $60^{\circ}$ and $90^{\circ}$ \\
Depth of tube center, $z_{0}$ & $34.5 \mathrm{~mm}\left(60^{\circ}\right), 39.5 \mathrm{~mm}\left(90^{\circ}\right)$ \\
Peak velocity, $v_{0}$ & $0.1 \mathrm{~m} / \mathrm{s}$ \\
\hline Measurement, STA processing & 401 \\
\hline Points in line, $N+1$ & $12.5 \mu \mathrm{m}$ \\
Spatial sampling interval, $\Delta x$ & $5 \mathrm{~mm}$ \\
Line length & $50^{\circ}$ \\
Angular sampling interval, $\Delta \theta$ & Linear regression $[10]$ \\
Stationary echo cancelling method & 113 \\
Number of correlations averaged, $L$ & $-1 \mathrm{to} 1 \mathrm{~m} / \mathrm{s}$ \\
Velocity search range & \\
\hline
\end{tabular}

where $E_{v=v^{\prime}}$ is the total energy of the 2-D HRI point spread function when the point moves at velocity $v^{\prime}$. The HRI point spread function is found from $-20 \mathrm{~mm}$ to $20 \mathrm{~mm}$ lateral and $20 \mathrm{~mm}$ to $40 \mathrm{~mm}$ axial for every $40 \mu \mathrm{m}$. This calculation of the point spread function energy assumes that the noise power is the same in both cases. $\triangle S N R$ is plotted in Fig. 2 as a function of the movement between LRIs given by $v T_{p r f} / \lambda$, where $T_{p r f}$ is the pulse repetition period. The same simulation is repeated for lateral movement at the same velocities. The resulting $\triangle S N R$ is plotted in Fig. 2 with a dashed line. The SNR during axial movement is significantly deteriorated at an inter-LRI movement of $\sim 0.25 \lambda$, whereas the SNR at $\sim 0.5 \lambda$ is comparable to that of a stationary scatterer.

From these simulations the lateral contrast at different velocities can also be extracted. This has been done, and the resulting plot is shown in Fig. 3 for purely axial motion. The results show the same tendency as [3]. For small velocities the contrast is almost unchanged, but for axial motion of $v^{\prime}=0.35$ $\mathrm{m} / \mathrm{s}$ in Fig. 3, corresponding to $0.25 \lambda$, the lateral side-lobes are up almost $20 \mathrm{~dB}$ compared to the stationary case. This is the worst case where the received echoes are $180^{\circ}$ out of phase, and looking at Fig. 2 also close to where the SNR is lowest. At higher axial velocities both contrast and SNR is better.

The motion compensation method is applied to the single point simulation data. Fig. 4 shows the lateral side-lobe 


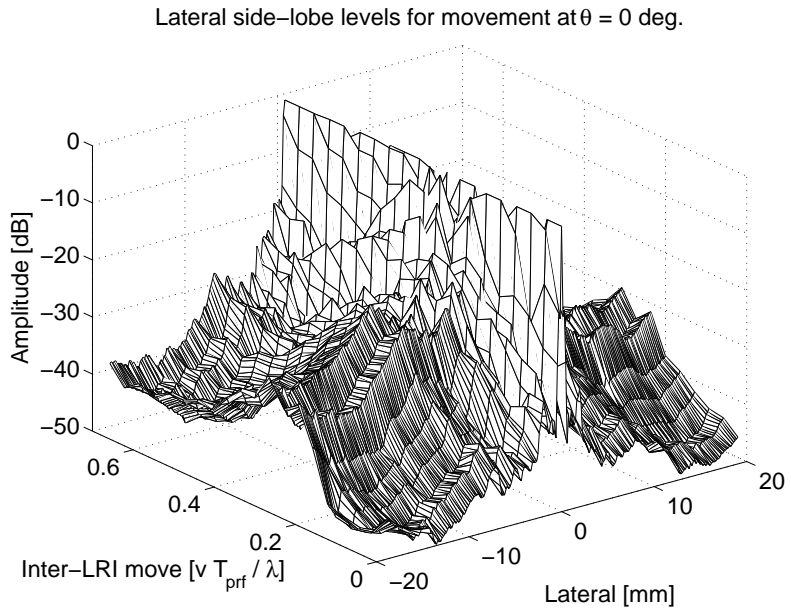

Fig. 3. Lateral side-lobe structure at different purely axial velocities. A $\sim 20$ $\mathrm{dB}$ increase in side-lobes is seen around $v=0.35 \mathrm{~m} / \mathrm{s}$ corresponding to a movement of $0.25 \lambda$ between LRIs.

Lateral side-lobe levels for movement at $\theta=0$ deg. after compensation

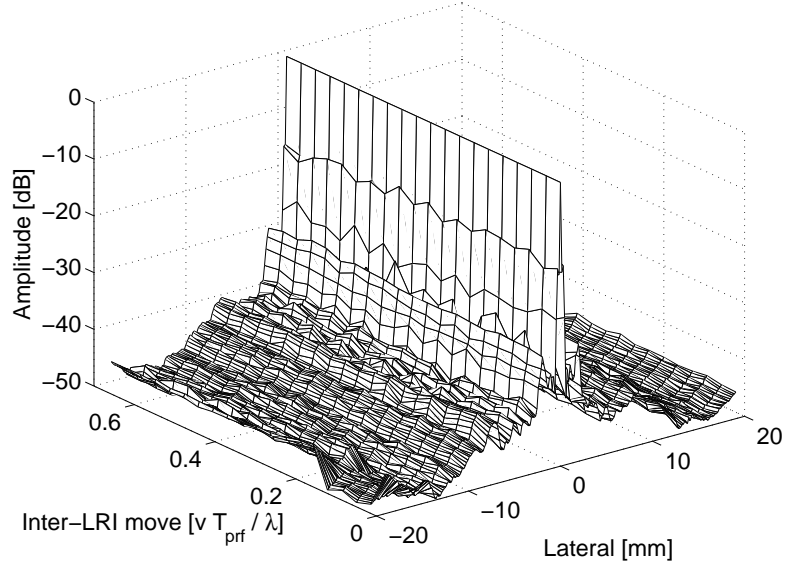

Fig. 4. Lateral side-lobe structure at different purely axial velocities when the motion is compensated in beamforming.

structure for the compensated simulations for purely axial motion. This can be compared to Fig. 3. After compensation almost no increase in side-lobe levels due to the motion is seen. This demonstrates the potential for the motion compensation.

\section{Phantom EXPERIMENTS}

A number of experiments were conducted using a flow rig phantom where blood mimicking fluid was circulated through tubes. A linear array transducer was fixed at a certain height $z_{0}$ above the tubing and at a certain angle $\theta$ with respect to the flow. The flow was constant over time, and the flow velocity profile was assumed parabolic. The velocity directly below the transducer center as a function of depth $z$ is then

$$
v(z)=v_{0}\left(1-\left(\frac{\left(z-z_{0}\right) \sin (\theta)}{R}\right)^{2}\right),\left|z-z_{0}\right|<\frac{R}{\sin (\theta)},
$$

where $\mathrm{R}$ is the vessel radius. Data were acquired using our RASMUS ultrasound multichannel sampling system [15] and processed as described in Section II. Various parameters for the data acquisition and processing are given in Table I.

Measurements were made for flow angles of $\theta=60^{\circ}$ and $90^{\circ}$ and for pulse repetition frequencies of $f_{p r f}=5 \mathrm{kHz}$ and $1 \mathrm{kHz}$. This corresponds to a peak movement of $\sim 7 \%$ and $35 \%$ of $\lambda$ between emissions with the peak velocity $v_{0}=0.1$ $\mathrm{m} / \mathrm{s}$ and $\lambda=0.28 \mathrm{~mm}$. Lowering the $f_{p r f}$ is equivalent to increasing the velocity.

Velocity directions and magnitudes were estimated along a profile through the tube at 20 uncorrelated time instances, and the estimated velocities were compared to the expected velocity profile given by (14). The resulting angle and velocity estimates for the $f_{p r f}=1 \mathrm{kHz}$ and $\theta=60^{\circ}$ experiment are shown in Fig. 5, which also show the average profiles \pm 3 times the standard deviation. A number of false angle estimates is seen mainly between 35 and $40 \mathrm{~mm}$ depth, resulting in too low velocity estimates.

The compensation method given by (12) is applied to the data prior to velocity estimation. Here the previous estimate of the vector velocity is used to compensate for the interLRI movement while beamforming. The resulting profiles are plotted in Fig. 6, where the majority of the false estimates are no longer present.

The average bias $\bar{B}_{v}$ and average standard deviation $\bar{\sigma}_{v}$ of the 20 estimated velocity profiles are used as a measure for the performance of the velocity estimation method. These are given by

$$
\begin{aligned}
& \bar{B}_{v}=\frac{1}{v_{0}\left(z_{2}-z_{1}\right)} \sum_{z=z_{1}}^{z_{2}}(\bar{v}(z)-v(z)) \\
& \bar{\sigma}_{v}=\frac{1}{v_{0}\left(z_{2}-z_{1}\right)} \sum_{z=z_{1}}^{z_{2}} \sqrt{\frac{1}{20} \sum_{i=1}^{20}\left(\hat{v}_{i}(z)-\bar{v}(z)\right)^{2}}
\end{aligned}
$$

where $z_{1}=z_{0}-R / \sin \theta$ and $z_{2}=z_{0}+R / \sin \theta$ are the tube edges, $\hat{v}_{i}(z)$ is the $i^{\text {th }}$ velocity estimate at depth $z$, $v(z)$ is the true velocity at depth $z$ as defined by (14), and $\bar{v}(z)=\sum_{i=1}^{20} \hat{v}_{i}(z) / 20$ is the average estimate at depth $z$. These quantities are calculated for the four experiments and are given in Table II.

TABLE II

AVERAGE BIAS AND STANDARD DEVIATION OF VECTOR VELOCITY ESTIMATES IN $\%$ OF PEAK VELOCITY $v_{0}$ FOR PROFILE MADE WITH AND WITHOUT COMPENSATION.

\begin{tabular}{llllll}
\hline Angle & $f_{p r f}$ & Bias & Std & Bias, comp & Std, comp \\
\hline $60^{\circ}$ & $5 \mathrm{kHz}$ & $-3.1 \%$ & $3.7 \%$ & $-2.8 \%$ & $3.7 \%$ \\
$60^{\circ}$ & $1 \mathrm{kHz}$ & $-8.4 \%$ & $12.2 \%$ & $-3.6 \%$ & $2.8 \%$ \\
$90^{\circ}$ & $5 \mathrm{kHz}$ & $-0.1 \%$ & $5.6 \%$ & $0.5 \%$ & $5.6 \%$ \\
$90^{\circ}$ & $1 \mathrm{kHz}$ & $-50.8 \%$ & $30.1 \%$ & $-38.5 \%$ & $20.3 \%$ \\
\hline
\end{tabular}

Table II shows a significant decrease in both average bias and average standard deviation for the measurement at $\theta=60^{\circ}$ and $f_{p r f}=1 \mathrm{kHz}$, and a moderate decrease for the $\theta=90^{\circ}$ and $f_{\text {prf }}=1 \mathrm{kHz}$ experiment. Only small changes are seen for 

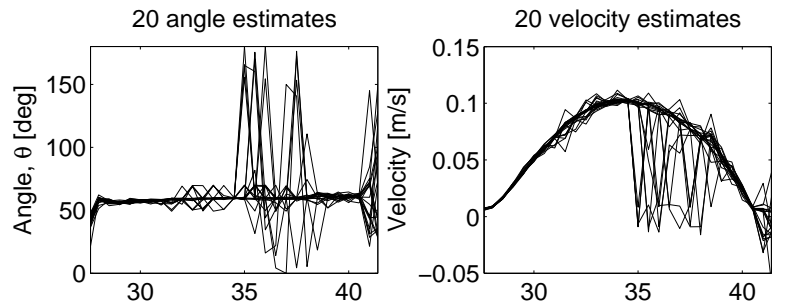

Mean \pm 3 standard deviation
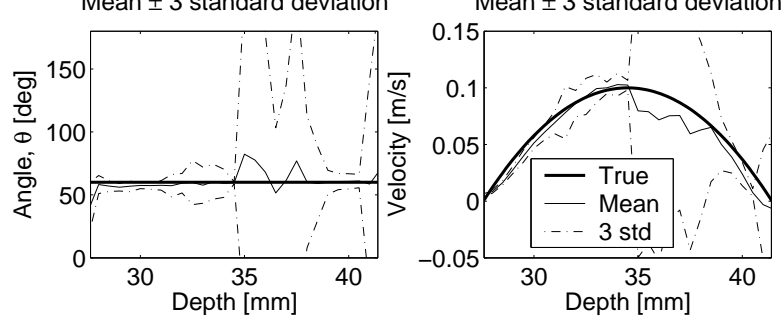

Fig. 5. Angle (left column) and velocity (right column) estimates for flow phantom experiments when no motion compensation is applied. The figure show 20 independent estimates (top) and the average and 3 times the standard deviation compared to the true values (bottom). The data are from the $\theta=60^{\circ}$ and $f_{p r f}=1 \mathrm{kHz}$ experiment.
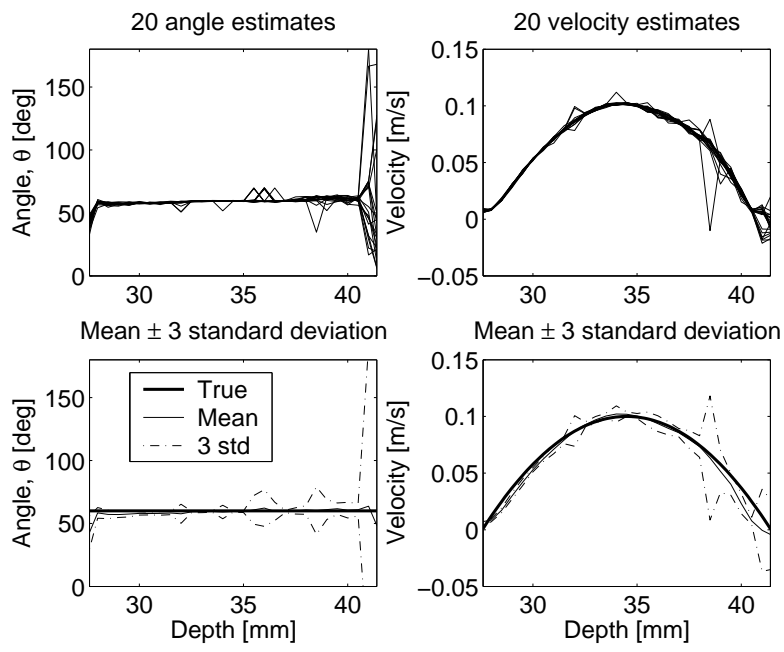

Fig. 6. Angle (left column) and velocity (right column) estimates for flow phantom experiments when the proposed motion compensation is applied. The figure show 20 independent estimates (top) and the average and 3 times the standard deviation compared to the true values (bottom). The data are from the $\theta=60^{\circ}$ and $f_{p r f}=1 \mathrm{kHz}$ experiment.

the other measurements. This indicates that the compensation method can decrease the number of false peaks when only a limited number of false peaks are present, and that the motion compensation has the largest effect at angles where the center frequency of the LRLs are highest.

\section{CONCLUSION}

A 2-D motion compensated beamforming for synthetic aperture vector flow imaging was presented, where the former velocity estimate was used in the beamforming of new data.

Simulations showed a large drop in signal-to-noise ratio and significantly increased lateral side-lobes when the axial inter-
LRI movement was around a quarter of a wavelength. The side-lobe increase was almost completely removed when the motion compensated beamforming was applied.

Flow phantom experiments of constant flow showed increased performance using the motion compensated beamforming on data where large movement is present. The method is yet to be tested on accelerating flow.

\section{ACKNOWLEDGEMENT}

This work was supported by grant 26-04-0024 from the Danish Science Foundation and by B-K Medical A/S, Herlev, Denmark.

\section{REFERENCES}

[1] G. E. Trahey and L. F. Nock. Synthetic receive aperture imaging with phase correction for motion and for tissue inhomogenities - part II: effects of and correction for motion. IEEE Trans. Ultrason., Ferroelec., Freq. Contr., 39:496-501, 1992.

[2] M. Karaman, H. Ş. Bilge, and M. O’Donnell. Adaptive multi-element synthetic aperture imaging with motion and phase aberation correction. IEEE Trans. Ultrason., Ferroelec., Freq. Contr., 42:1077-1087, 1998.

[3] C. R. Hazard and G. R. Lockwood. Effects of motion artifacts on a synthetic aperture beamformer for real-time 3D ultrasound. In Proc. IEEE Ultrason. Symp., pages 1221-1224, 1999.

[4] J. S. Jeong, J. S. Hwang, M. H. Bae, and T. K. Song. Effects and limitations of motion compensation in synthetic aperture techniques. In Proc. IEEE Ultrason. Symp., pages 1759 -1762, 2000.

[5] S. I. Nikolov and J. A. Jensen. K-space model of motion artifacts in synthetic transmit aperture ultrasound imaging. In Proc. IEEE Ultrason. Symp., pages 1824-1828, 2003.

[6] S. I. Nikolov and J. A. Jensen. Velocity estimation using synthetic aperture imaging. In Proc. IEEE Ultrason. Symp., pages 1409-1412, 2001.

[7] S. I. Nikolov and J. A. Jensen. In-vivo synthetic aperture flow imaging in medical ultrasound. IEEE Trans. Ultrason., Ferroelec., Freq. Contr., pages 848-856, 2003.

[8] J. A. Jensen. Velocity vector estimation in synthetic aperture flow and B-mode imaging. In IEEE International Symposium on Biomedical imaging from nano to macro, pages 32-35, 2004.

[9] K. L. Gammelmark and J. A. Jensen. Duplex synthetic aperture imaging with tissue motion compensation. In Proc. IEEE Ultrason. Symp., pages 1569-1573, 2003.

[10] A. P. G Hoeks, J. J. W. van de Vorst, A. Dabekaussen, P. J. Brands, and R. S. Reneman. An efficient algorithm to remove low frequency Doppler signal in digital Doppler systems. Ultrason. Imaging, 13:135145, 1991a.

[11] S. G. Foster. A pulsed ultrasonic flowmeter employing time domain methods. PhD thesis, Dept. Elec. Eng., University of Illinois, Urbana, Ill., 1985.

[12] J. A. Jensen and N. B. Svendsen. Calculation of pressure fields from arbitrarily shaped, apodized, and excited ultrasound transducers. IEEE Trans. Ultrason., Ferroelec., Freq. Contr., 39:262-267, 1992.

[13] J. A. Jensen. Field: A program for simulating ultrasound systems. Med. Biol. Eng. Comp., 10th Nordic-Baltic Conference on Biomedical Imaging, Vol. 4, Supplement 1, Part 1:351-353, $1996 \mathrm{~b}$.

[14] F. Gran and J. A. Jensen. Designing waveforms for temporal encoding using a frequency sampling method. IEEE Trans. Ultrason., Ferroelec., Freq. Contr., Submitted, 2006.

[15] J. A. Jensen, O. Holm, L. J. Jensen, H. Bendsen, S. I. Nikolov, B. G. Tomov, P. Munk, M. Hansen, K. Salomonsen, J. Hansen, K. Gormsen, H. M. Pedersen, and K. L. Gammelmark. Ultrasound research scanner for real-time synthetic aperture image acquisition. IEEE Trans. Ultrason., Ferroelec., Freq. Contr., 52 (5), May 2005. 